\title{
Symmetry of chaotic attractors and kink-antikink structures in CMLs
}

\author{
Guowei He, Jiachun Li \\ Laboratory for Nonlinear Mechanics of Continuous Media, Institute of Mechanics, Chinese Academy of Sciences, Beijing 100080, China
}

Received 4 June 1993; revised manuscript received 3 December 1993; accepted for publication 6 December 1993

Communicated by A.R. Bishop

\begin{abstract}
In this paper the symmetries of coupled map lattices (CMLs) and their attractors are investigated by group and dynamical system theory, as well as numerical simulation, by means of which the kink-antikink patterns of CMLs in space-amplitude plots are discussed.
\end{abstract}

\section{Introduction}

Spatiotemporal chaos in spatially extended systems, namely space-time disordered states, has become an extremely attractive topic due to its potential applications to turbulence, pattern formation in nature. As a simple model of spatiotemporal chaos in extended systems, the coupled map lattices [1] (CMLs) of the following form,

$u_{n+1}^{k}=(1-\epsilon) f\left(u_{n}^{k}\right)+\frac{1}{2} \epsilon\left[f\left(u_{n}^{k+1}\right)+f\left(u_{n}^{k-1}\right)\right]$,

$u_{n}^{0}=u_{n}^{N}, \quad u_{n}^{1}=u_{n}^{N+1}, \quad k=1,2, \ldots, N$,

have been intensively studied, where $u_{n}^{i} \in[-1,1]$ are the state variables associated with the $i$ th lattice point at the $n$th discrete time step, and $N$ is the system size or dimension. The parameter $\epsilon \in\left[0, \frac{1}{2}\right]$ in $(1)$ is called the coupling coefficient reflecting diffusive effects. The local mapping function $f(x)$ may be chosen as the logistic map: $f(x)=1-a x^{2}$, where the parameter $a$ is the nonlinear factor, $0 \leqslant a \leqslant 2,0 \leqslant x \leqslant 1$. Numerical simulations [2] show that CMLs display a variety of spatiotemporal complex phenomena, includ- ing spatiotemporal periodic, quasiperiodic and chaotic motions in different parameter ranges of $a$ and $\epsilon$. Although spatiotemporal patterns in CMLs are rather complicated, their orbits in phase spaces often retain some kind of geometric symmetries owing to the symmetries of the CMLs themselves. The bifurcations in CMLs are always accompanied with an alteration of the symmetries of their orbits in the phase spaces. The increase or decrease of the symmetries in bifurcated orbits is referred to as a symmetryincreasing bifurcation or symmetry-breaking bifurcation [3]. In this paper, the so-called $\mathrm{D}_{N}$-symmetry of CMLs and the associated symmetry of chaotic attractors in phase spaces or the spatial return map are carefully investigated. Based on the symmetry argument, the kink-antikink patterns in physical spaces are further examined.

\section{2. $D_{n}$-symmetry coupled map lattices}

To begin with, we rewrite (1) in the following equivalent form, 


$$
\begin{aligned}
& U_{n+1} \equiv\left(\begin{array}{c}
u_{n+1}^{1} \\
u_{n+1}^{2} \\
\vdots \\
u_{n+1}^{N}
\end{array}\right) \\
& =\left(\begin{array}{cccccc}
1-\epsilon & \frac{1}{2} \epsilon & 0 & \ldots & \frac{1}{2} \epsilon \\
\frac{1}{2} \epsilon & 1-\epsilon & & & 0 \\
0 & & \ddots & & \vdots \\
\vdots & & & & \\
& & & & \\
& & & & \frac{1}{2} \epsilon \\
\frac{1}{2} \epsilon & 0 & \ldots & & \frac{1}{2} \epsilon & 1-\epsilon
\end{array}\right)\left(\begin{array}{c}
f\left(u_{n}^{1}\right) \\
f\left(u_{n}^{2}\right) \\
\vdots \\
f\left(u_{n}^{N}\right)
\end{array}\right) \\
& \equiv E F\left(U_{n}\right) \text {, }
\end{aligned}
$$

where $E$ is the coefficient matrix and $F$ is the nonlinear vector operator.

Let $\mathrm{D}_{N}=\left\{I, T, T^{2}, \ldots, T^{N-1}, K\right\}$ be the dihedral group, where

$I=\left(\begin{array}{cccc}1 & 0 & \ldots & \\ 0 & 1 & & \\ \vdots & & \ddots & \\ & & & \\ & & & 1\end{array}\right)$,

$T=\left(\begin{array}{ccccc}0 & 1 & 0 & \ldots & \\ 0 & 0 & 1 & & \\ \vdots & & \ddots & \ddots & \\ & & & & \\ & & & & 1 \\ 1 & 0 & \ldots & & 0\end{array}\right)$,

$K=\left(\begin{array}{llll} & & 0 & 1 \\ & & 1 & 0 \\ & . . & & \\ 1 & & & \end{array}\right)$.

It can be readily shown that the following commutation operations hold even for nonlinear operators $F$ since for each element $\sigma$ in $\mathrm{D}_{N}$ it merely implies the change of order,

$\sigma E=E \sigma$.

Then

$\sigma U_{n+1}=\sigma E F\left(U_{n}\right)=E \sigma F\left(U_{n}\right)=E F\left(\sigma U_{n}\right)$.

Therefore, CML (1) is $\mathrm{D}_{N}$-symmetric or equivariant.

\section{Symmetry of chaotic attractors in CMLs}

As indicated in the previous section, the CML is $\mathrm{D}_{n}$-symmetric. In this section, the geometric symmetry of its attractors in phase spaces and its influences on patterns in physical spaces are further investigated.

Let $A$ be an invariant set in the CML, that is $A=E F(A)$, then the set $\sigma A$ is also invariant. As a matter of fact, $\sigma A=\sigma E F(A)=E \sigma F(A)=E F(\sigma A)$. We may as well call $\sigma A$ a conjugate set of $A$.

If $A$ is a regular attractor, that is, $A$ represents a fixed point, periodic or quasiperiodic orbits, $\sigma A$ is also a regular attractor. Now assuming the intersection of $A$ and $\sigma A$ is nonempty, as shown by numerical simulation, we conclude that $A$ and $\sigma A$ are the same attractor according to the uniqueness of orbits in CMLs. Therefore $A$ is $\sigma$-symmetric. Especially, $A$ is $\mathrm{D}_{n}$-symmetric if $\sigma A=A$ for any $\sigma \in \mathrm{D}_{n}$

If $A$ is a chaotic attractor, that is, the closure of all the unstable periodic orbits [4], and the set $B$ consists of all the unstable periodic orbits in the CML, then $B \in A$. Notice that $\sigma B$ is also the set of unstable periodic orbits in the CML, so we obtain $\sigma B \in A$. As a result, the closure $\sigma A$ of $\sigma B$ belongs to $A$, which means that $A$ and $\sigma A$ intersect. According to the uniqueness of chaotic attractors in deterministic dynamical systems, $\sigma A$ and $A$ must be the same invariant set. Now it is shown that the chaotic attractors of the CML are $\mathrm{D}_{n}$-symmetric or they keep all the symmetries of the CML.

Define the projection operator $P_{i j}$ satisfying $P_{i j} u=$ $\left(u_{i}, u_{j}\right)^{\mathrm{T}}$. The flip $K$ restricted on the subspace $P_{i j} A=\left\{\left(u_{i}, u_{j}\right)^{\mathbf{T}}\right\}$ can be represented as

$K=\left(\begin{array}{ll}0 & 1 \\ 1 & 0\end{array}\right)$.

It is readily verified that

$K\left(P_{i j} A\right)=P_{i j} A$,

in which

$K\left(P_{i j} A\right)=\left\{\left(u_{j}, u_{i}\right)^{\mathrm{T}}:\left(u_{i}, u_{j}\right)^{\mathrm{T}} \in P_{i j} A\right\}$.

In fact, for every $\left(u_{i}, u_{j}\right)^{\mathrm{T}} \in P_{i j} A$, there always exists a $u \in A$ such that $P u=\left(u_{i}, u_{j}\right)^{\mathrm{T}}$. Then $K u \in K A=A$. Making the projection $P_{i j}$ on the above equation, we have $P_{i j}(K u)=\left(u_{j}, u_{i}\right)^{\mathrm{T}} \in P_{i j} A$, 

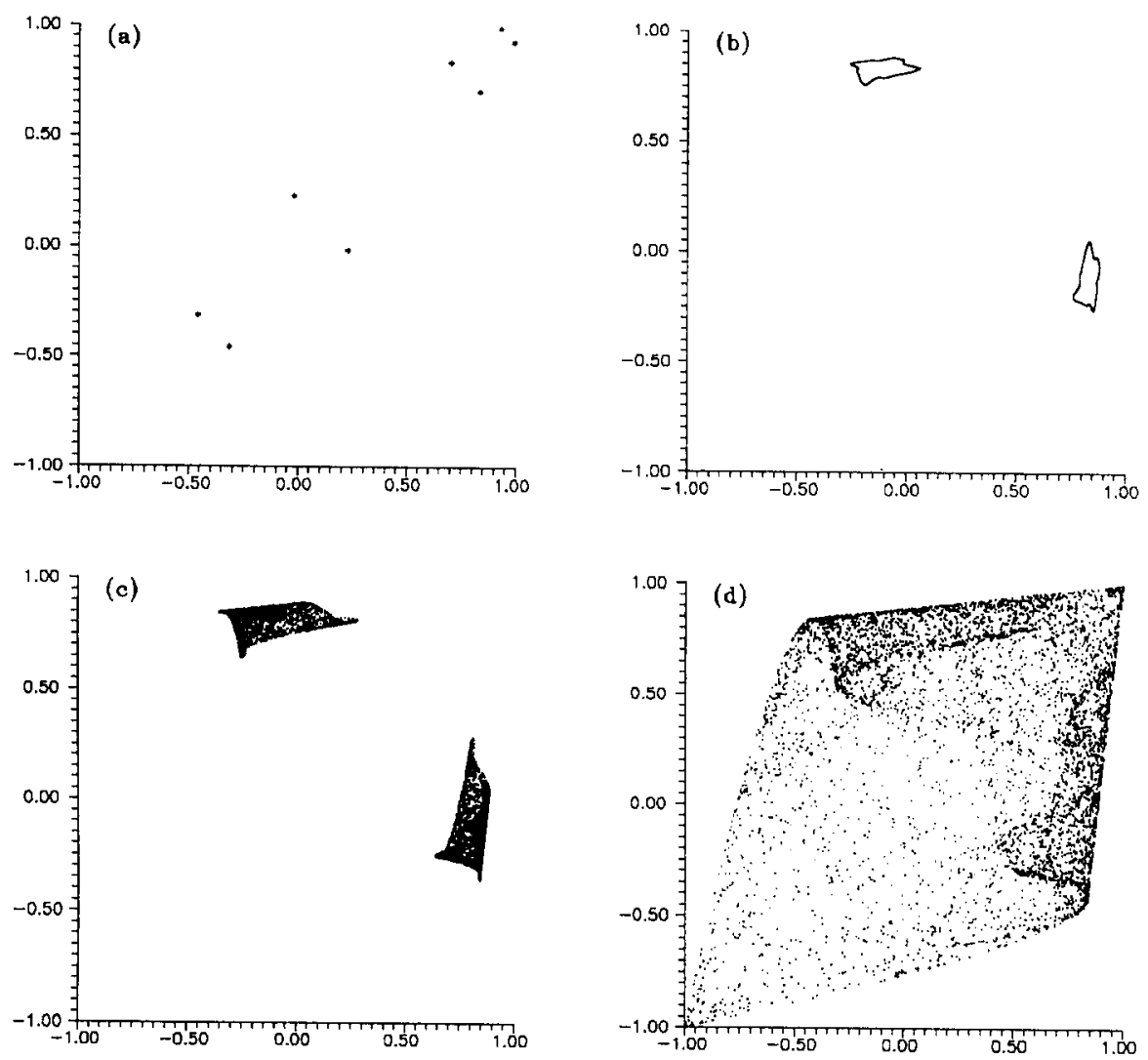

Fig. 1. Phase diagrams with $N=2$. (a) $a=1.5$, (b) $a=1.8$, (c) $a=1.9$, (d) $a=2.0$.

and multiply by $K$

$K\left(u_{j}, u_{i}\right)^{\mathrm{T}}=\left(u_{i}, u_{j}\right)^{\mathrm{T}} \in K\left(P_{i j} A\right)$,

which means $P_{i j} A \in K\left(P_{i j} A\right)$. For the same reason, we can verify $K\left(P_{i j} A\right) \in\left(P_{i j} A\right)$. Therefore (4) holds.

(4) shows that the projection of the invariant set $A$ on the subspace $P_{i j} A$ is $\mathrm{D}_{2}$-symmetric in which $\mathrm{D}_{2}=\{I, K\}$. Especially, we have $P_{i, i+1} u=\left(u_{i}, u_{i+1}\right)^{\mathrm{T}}$ for the spatial return map such that the attractors of the CML are symmetric about the diagonal line of the first and third quadrants, which theoretically verifies the numerical results given by Kaneko [2].

As an example, the CML with $N=2$ is given by

$u_{n+1}^{1}=(1-\epsilon) f\left(u_{n}^{1}\right)+\epsilon f\left(u_{n}^{2}\right)$,

$u_{n+1}^{2}=(1-\epsilon) f\left(u_{n}^{2}\right)+\epsilon f\left(u_{n}^{1}\right)$,

which is $\mathrm{D}_{2}$-symmetric. According to the above results, its chaotic attractors are symmetric about the diagonal line of the first and third quadrants. $\mathrm{Nu}$ - merical calculations have been conducted for $\epsilon=0.1$, and $a=1.5,1.8,1.9,2.0$. The attractors in the CML evolve from the symmetric periodic points to ears, and finally to the black rhombus about the diagonal line of the first and third quadrants (see Figs. la1d). The $D_{2}$-symmetry suggests that there must exist states $\left(u_{i}, u_{i+1}\right)^{\mathrm{T}}$ if there exist states $\left(u_{i+1}, u_{i}\right)^{\mathrm{T}}$ in the CML, which is responsible for the various kinds of kink-antikink structures in space-amplitude plots for the CML.

\section{Conclusion}

We have discussed the symmetries of the CML and its attractors by numerical simulation and theoretical analysis. It is found that the CML is $\mathrm{D}_{n}$-symmetric itself, and these symmetries are retained by the attractors of the CML such that the orbits in spatial return maps are symmetric about the diagonal line of 
the first and third quadrants. The kink-antikink patterns of CMLs in space-amplitude plots stem from just this kind of symmetry about the diagonal line. The above arguments are applicable for the CML (1) with the nonlinearly coupled terms like $f(x)=$ $1-a x^{2}$, as well as for the coupled maps with linearly coupled terms like Eq. (1.1) in Ref. [5]. since their coupling forms are $\mathrm{D}_{n}$-symmetric. The classification of patterns of CMLs with symmetry based on group theory is a useful method to study spatiotemporal chaos.

\section{References}

11] I.P. Crutchficld and K. Kaneko, Phenomenology of spatiotemporal chaos, in: Directions in chaos (World Scientific, Singapore. 1987 !

[2] K. Kaneko. Physica D 34 (1989),

[3] P. Chossat and M. Golubitsky, Physica D 32 (1988) 423.

[4] P. Collet and J,-P. Eckmann, Iterated maps on the interval as dynamical systems (Birkhauser, Basel, 1980).

[5] K. Kaneko. Prog. Theor. Phys. 69. No. 5 (1983). 